\title{
Biogeografía del tomate Solanum lycopersicum var. cerasiforme (Solanaceae) en su centro de origen (sur de América) y de domesticación (México)
}

\author{
Gino Délices ${ }^{1}$, Otto Raúl Leyva Ovalle ${ }^{1}$, Claudio Mota-Vargas ${ }^{2}$, Rosalía Núñez Pastrana ${ }^{1}$, \\ Roberto Gámez Pastrana ${ }^{1}$, Pablo Andrés Meza ${ }^{1} \&$ Ricardo Serna-Lagunes ${ }^{1}$ \\ 1. Facultad de Ciencias Biológicas y Agropecuarias, Región Orizaba-Córdoba, Universidad Veracruzana. Peñuela, SN, \\ Col. Centro, 94945 Amatlán de los Reyes Veracruz, México; ginodelices@gmail.com, oleyva@uv.mx, \\ ronunez@uv.mx,mgamez@uv.mx,pandres@uv.mx,rserna@uv.mx \\ 2. Instituto de Ecología A. C., camino Antiguo a Coatepec 351, El Haya, 91070 Xalapa Enríquez, Veracruz, México; \\ claudio.mota@inecol.mx \\ * Correspondencia
}

Recibido 28-XI-2018. Corregido 06-II-2019. Aceptado 05-VIII-2019.

\begin{abstract}
Biogeography of tomato Solanum lycopersicum var. cerasiform (Solanaceae) in its center of origin (South America) and domestication (Mexico). The tomato (Solanum lycopersicum) is one of the main horticultural crops in the world because of the important economic benefits that its commercialization generates. Even though the species is widely cultivated in the world, it is susceptible to pests, diseases, and environmental stresses due to the loss of its genetic diversity. There is a wild form called $S$. $l$. var ceraciforme that is distributed from South America (its center of origin) to Mexico (its area of domestication), specifically on the slope of the Gulf of Mexico in Veracruz. Due to the large genetic diversity of these wild tomatoes, it is important to improve its crop. The objective of this investigation was to identify patterns of environmental diversification of the tomato, infer the processes of adaptation of the populations in the area of domestication with respect to their center of origin and propose measures of conservation and variation of $S$. l. var. cerasiforme. We generated two ecological niche models (MNE) with Maxent version 3.4.1 (evaluated with AUC and partial-ROC) to determine the suitability of environmental conditions including their respective projections from the domestication center to the area of origin and vice versa. We used eight variables of temperature and precipitation. Additionally, we included tomato records from bibliographical sources and fieldwork. We also used the niche comparison test (equivalency and similarity) proposed by Broennimann et al. (2012) to evaluate the similarity of environmental conditions in both regions. Subsequently, we carried out an analysis of variance followed by a mean comparison test (Tukey, $\mathrm{P} \leq 0.05)$ with all environmental variables measured. The values of AUC $(0.93$ and 0.80$)$ and partial-ROC $(1.86$ and $1.71, \mathrm{P}=0.0001)$ of the MNE showed that they were good predictive models. We observed that, in the center of Mexico, there are environmental conditions similar to those of the center of origin, unlike the slope of the Gulf of Mexico where they are different. The equivalency test showed that the environmental comparison between both regions is less similar than expected by chance $(\mathrm{P}=0.003)$. The similarity test indicated that the existing similarity between both regions can also be obtained by chance $(\mathrm{P}=0.683)$. We also found significant differences in three temperature and precipitation variables. In conclusion, we determined that the center of origin and domestication of $S$. $l$. var. cerasiforme has similar environmental characteristics despite the geographic distance; nevertheless, there are geographical zones (the Gulf of Mexico in Veracruz) in the area of domestication with different environmental conditions. Those places have the potential to contain valuable germplasms.
\end{abstract}

Key words: distribution; ecological niche modeling; environmental predictors; center of origin; domestication area; comparison of ecological niche.

Délices, G., Leyva Ovalle, O. R., Mota-Vargas, C., Núñez Pastrana, R., Gámez Pastrana, R., Meza, P. A., \& Serna-Lagunes, R. (2019). Biogeografía del tomate Solanum lycopersicum var. cerasiforme (Solanaceae) en su centro de origen (sur de América) y de domesticación (México). Revista de Biología Tropical, 67(4), 1023-1036. 
El tomate (Solanum lycopersicum) es una de las principales hortalizas en el mundo por su demanda en el mercado y las grandes ganancias económicas que genera su comercialización (FAOSTAT, 2016). Esta especie es una planta arbustiva que, en su forma silvestre, puede tener un ciclo de vida superior a un año; sin embargo, con la ayuda de la ingeniería genética y procesos de selección, se han desarrollado cultivares que, con una infraestructura adecuada para controlar las condiciones ambientales de cultivo - así como el uso de insumos como fertilizantes, insecticidas, herbicidas- permiten obtener mejores rendimientos en poco tiempo.

A pesar de que la especie es ampliamente cultivada en el mundo, su diversidad genética se considera restringida (Peralta, Knapp \& Spooner 2005; Govindaraj, Vetriventhan, \& Srinivasan, 2015; Rao \& Hodgkin, 2002). Como consecuencia, son más susceptibles a plagas, enfermedades, estrés abiótico, así como a la pérdida de sus atributos sensoriales (Bolger, Lohse, \& Usadel, 2014). Este rasgo hace que las poblaciones silvestres de tomate representen una fuente de genes que pueden conferir resistencia o tolerancia a plagas y enfermedades en las variedades actualmente cultivadas (Álvarez-Hernández, Cortez-Madrigal, \& García-Ruiz, 2009; BonillaBarrientos et al., 2014).

Existe una forma silvestre $S$. $l$. var ceraciforme que es de particular importancia, ya que se considera el ancestro de los cultivares que actualmente existen en el mundo (SánchezPeña et al., 2006; Peralta \& Spooner, 2007). Esta se distribuye desde América del Sur en países - como Perú, Ecuador, Chile e Islas Galápagos - (Peralta \& Spooner, 2007; Nakazato, Warren, \& Moyle, 2010) y continúa por toda América Central hasta México (Ranc, Muños, Santoni, \& Causse, 2008; Flores-Hernández et al., 2017). Esta zona se ha considerado el centro de domesticación, particularmente en la vertiente del Golfo de México en los estados de Veracruz y Puebla (Long, 1995). En este proceso de dispersión desde el sur de América hasta México (Nakazato et al., 2010; Jenkins, 1948), la especie pudo establecerse en áreas con condiciones climáticas similares a las de su origen, pero también pudo adaptarse a condiciones climáticas distintas (Croteau, 2010), lo que implica que la especie tuvo que modificar sus requerimientos fisiológicos y características morfológicas en respuesta a las nuevas condiciones ambientales (Nakazato et al., 2010; Khimoun et al., 2013).

Para comparar dos ambientes en donde se ha desarrollado una especie, como lo es el centro de origen y el de domesticación de $S$. l. var ceraciforme, Broennimann et al. (2012) propusieron un análisis multivariado en donde se determina si las variables ambientales de dos regiones en particular son diferentes o similares entre sí, con base en un índice de similitud de Schoener (1968). Mediante la propiedad intrínseca de los individuos de una especie de adaptarse a diferentes condiciones ambientales, la cual está ligada a la variabilidad geográfica y ambiental en la que se desarrolla (Hoyt, 1992; Rao \& Hodgkin, 2002; Zuriaga et al., 2009), es posible inferir si existe una diversificación de los patrones de distribución ambiental en la región de domesticación con respecto a su centro de origen del tomate.

Existen poblaciones de tomate en el área de domesticación cuyas condiciones ambientales podrían ser distintas a las de su centro de origen. El centro de Veracruz, por ejemplo, es de particular importancia, ya que allí se encuentran poblaciones en una gran variedad de ambientes y ecosistemas, lo que sugiere que varias se han adaptado y potencialmente representarían material fundamental como banco de germoplasma. En el presente trabajo, se caracterizan y comparan las condiciones ambientales de ambas regiones (origen y domesticación) para identificar los patrones de diversificación ambiental, así como determinar las áreas de distribución geográfica potencial de la especie en donde se podrían establecer medidas de conservación y rescate del tomate S. l. var ceraciforme.

\section{MATERIALES Y MÉTODOS}

Registros de presencia de $S$. $l$. var ceraciforme: Para identificar las áreas de distribución 
geográfica potencial del centro de origen y de domesticación de $S$. l. var ceraciforme, se generaron modelos de nicho ecológico (MNE). Para generar estos modelos se requiere de dos fuentes de información: los registros de la especie y coberturas o variables ambientales. Por ello, se recopilaron registros de presencia de $S$. $l$. var. cerasiforme en la bibliografía especializada (Arce, 2015; Álvarez-Hernández et al., 2009), la base de datos de la Infraestructura Global de Información sobre Biodiversidad (GBIF, 2018) y el trabajo de campo (como se describe más adelante).

Posteriormente, se realizó una base de datos con todos los registros disponibles: se eliminaron los registros duplicados que carecieran de precisión en la localidad de registro o que cayeran dentro de una misma celda o pixel $\left(\sim 1 \mathrm{~km}^{2}\right)$ equivalente al tamaño (resolución) de las variables utilizadas para generar los modelos (como se detalla posteriormente). Esto se realizó con la ayuda de los Sistemas de Información Geográfica (SIG). Mediante este proceso, se obtuvo un total de 98 registros de S. l. var. cerasiforme para el centro de origen y 67 para el área de domesticación. En ambos casos se utilizó el $80 \%$ de los puntos para generar los modelos; el otro $20 \%$ se utilizó para validarlo con base en la Roc Parcial como se describe posteriormente.

Muestreo de áreas seleccionadas: El trabajo de campo se realizó en el norte, centro y sur del estado de Veracruz, México de septiembre 2016 a noviembre 2017 (Tabla 1, Fig. 1A, Fig. 1B). Considerando que $S$. $l$. var. cerasiforme se distribuye desde el nivel del mar hasta los 3300 msnm (Álvarez-Hernández et al., 2009), los recorridos en campo se realizaron entre dicho rango altitudinal. En cada localidad, se registró la ubicación geográfica y topográfica con un GPS marca Garmin y se identificó el tipo de vegetación dominante en el área con la finalidad de reportar la vegetación asociada al tomate. Además, se obtuvo el perfil climático de temperatura y precipitación (con base en las variables ambientales que se describen a continuación) para poder caracterizar ambientalmente y de manera puntual los registros obtenidos en campo.

Las variables ambientales se obtuvieron del proyecto Worldclim, el cual proporciona valores promedio para 1950-2000 (Hijmans, Cameron, Parra, Jones, \& Jarvis, 2005) con una resolución espacial de 1 pixel $=0.0083^{\circ}$ $\left(\sim 1 \mathrm{~km}^{2}\right)$. Para elegir las variables que se utilizaron para correr los modelos, primero se evaluó la multicolinealidad entre las 19 variables con base en un análisis de coeficiente de correlación (r) (Pearson \& Dawson, 2003). Posteriormente, se determinó como umbral de corte un valor de correlación de $r \geq 0.80$. Finalmente, se eliminaron las variables que estaban altamente correlacionadas (variables con un valor de $r \geq 0.80$; Yang, Kushwaha, Saran, Xu, \& Roy, 2013).

Este proceso se realizó con la finalidad de evitar la autocorrelación espacial entre las variables (Escobar, Qiao, Phelps, Wagner, \& Larkin, 2016), lo cual puede provocar un sobreajuste en la construcción del modelo (Lozier, Aniello, \& Hickerson, 2009). Las ocho variables que se utilizaron para correr los modelos fueron: biol (temperatura media anual, ${ }^{\circ} \mathrm{C}$ ), bio2 (rango medio diurno de temperatura, ${ }^{\circ} \mathrm{C}$ ), bio3 (isotermalidad, ${ }^{\circ} \mathrm{C}$ ), bio4 (índice de variación estacional de temperatura), bio12 (precipitación media anual, $\mathrm{mm}$ ), bio14 (precipitación del mes más seco, mm), bio15 (índice de variación estacional de la precipitación) y bio18 (precipitación del trimestre más seco, mm).

Construcción de los modelos: Los modelos se generaron con el algoritmo Maxent versión 3.4.1 que se basa en el principio de máxima entropía para determinar la idoneidad de las condiciones ambientales para la especie (Phillips, Anderson, \& Schapire, 2006). Este algoritmo ha sido ampliamente utilizado y ha demostrado un buen desempeño (Elith et al., 2006). Maxent se configuró por defecto (Phillips \& Dudik, 2008), se desactivaron los módulos de "extrapolate" y "do Clamping" y se utilizó un formato de salida logístico para el modelo. Los parámetros establecidos fueron 


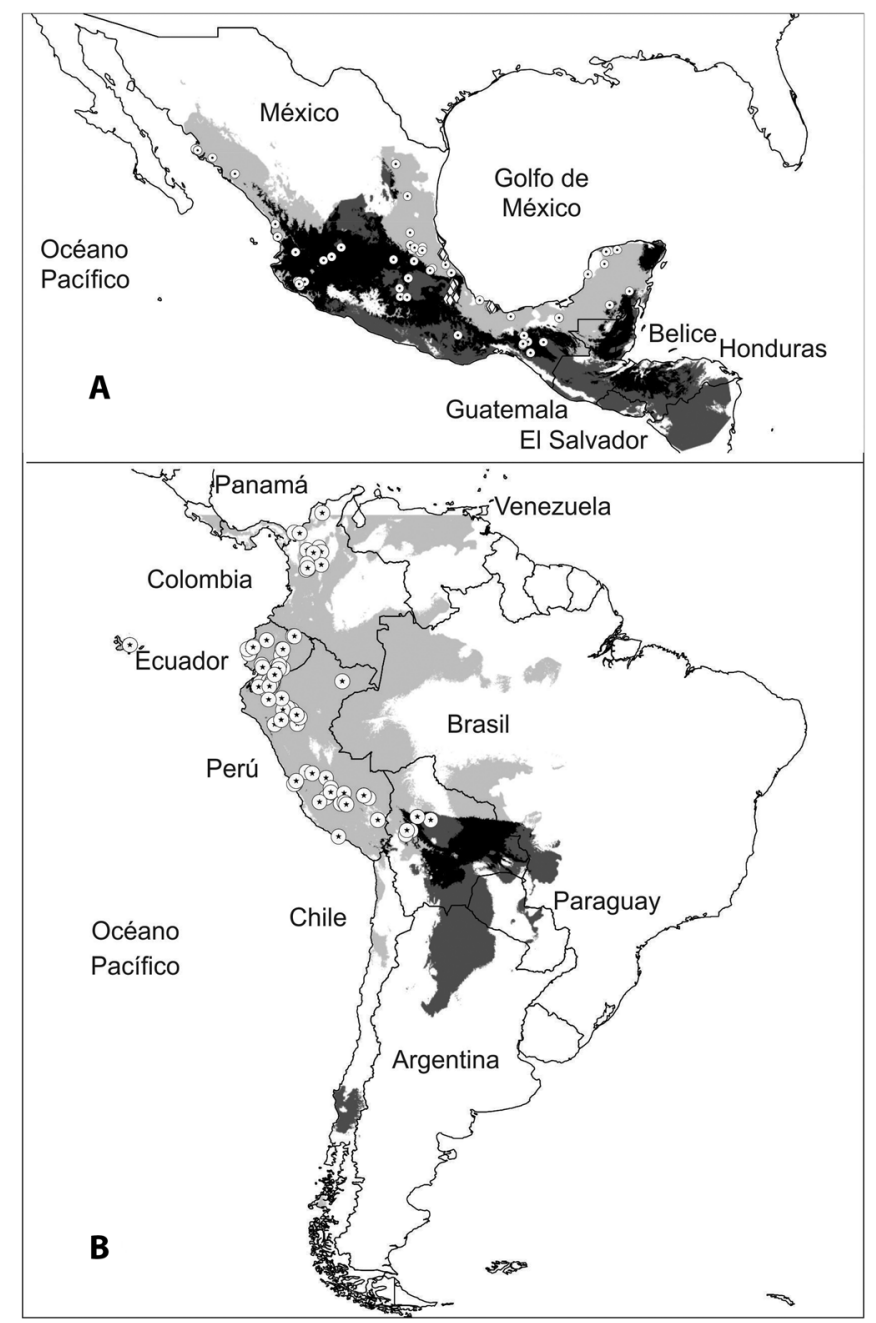

Fig. 1. Modelo de nicho ecológico de $S$. $l$. var. cerasiforme en México (A) y el sur de América (B). En ambos casos, los puntos en blanco representan los registros históricos. En A, los diamantes representan los registros en campo. El color gris claro representa el MNE en cada región; en gris oscuro la proyección de México (A) hacia el sur de América (B) y viceversa; en negro la superposición de cada región.

Fig. 1. Ecological niche model of S. $l$. var. cerasiforme in Mexico (A) and South America (B). In both cases, the blank points show the historical records. In A, the diamonds represent the records in the field. The light gray color represents the MNE in each region; in dark gray the projection from Mexico (A) to the south of America (B) is shown and vice versa; in black the overlap of each region is shown.

los siguientes: un valor máximo de 1000 iteraciones, un límite de convergencia de 0.00001 y el valor del factor de regularización en 1 .

Seguidamente, se generaron dos MNE y sus correspondientes proyecciones, es decir, se corrió el MNE en México que representa el área de domesticación y se proyectó en Suramérica (que representa el centro de origen). Posteriormente, se generó un MNE en Suramérica (origen) y se proyectó a México (domesticación) con la finalidad de identificar la superposición geográfica de las condiciones ambientales en ambas regiones. Para correr los modelos, se delimitó el área de accesibilidad o "M" (sensu Soberón \& Peterson, 2005) con base en las provincias biogeográficas definidas 


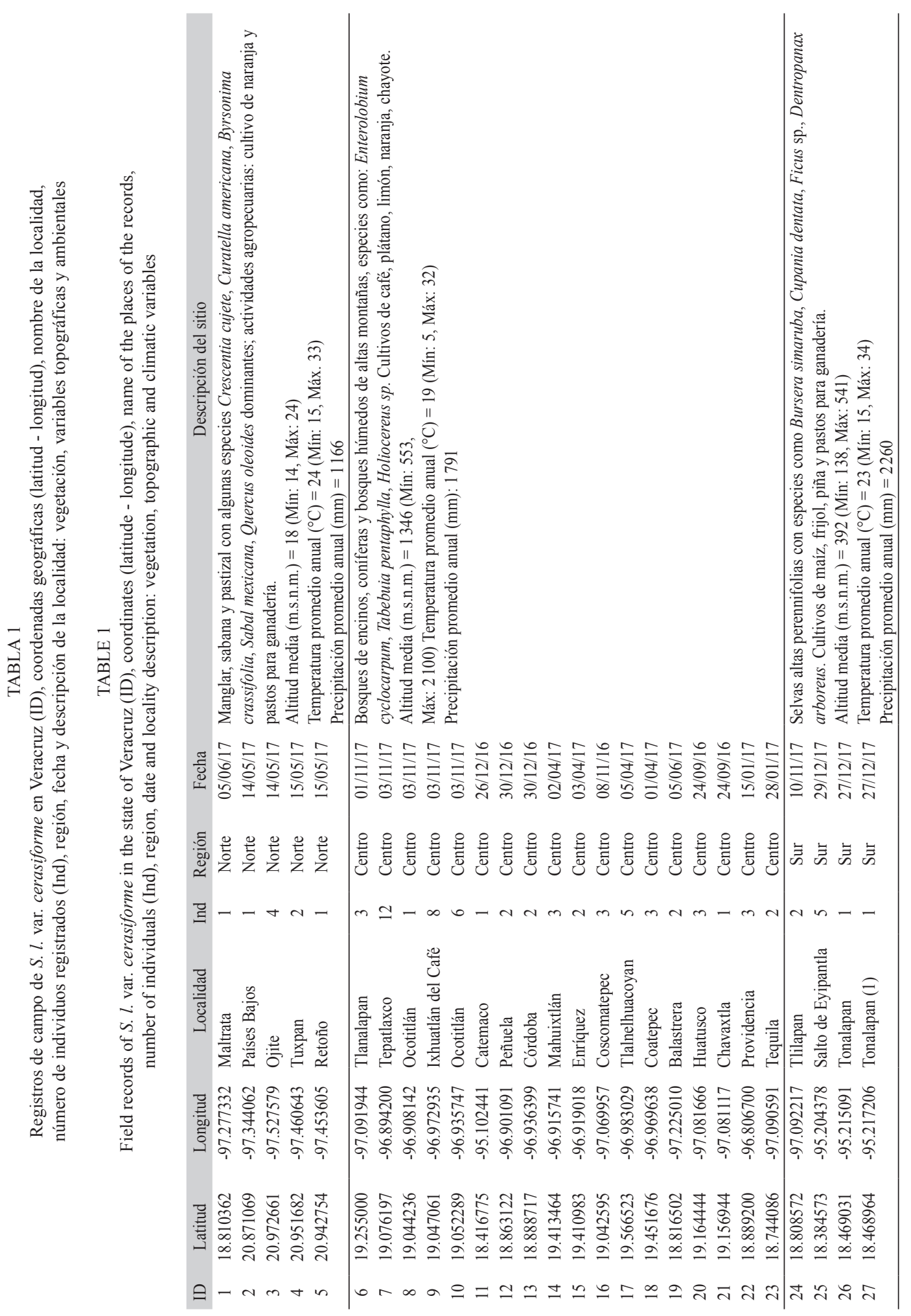


por Morrone (2014) y Löwenberg-Neto (2014). Específicamente, en el centro de origen se incluyó la región Neotropical formada por los países de Panamá, Venezuela, Colombia, Ecuador, Perú, Bolivia, Chile, Argentina Paraguay y Brasil. Para el caso del área de domesticación, la "M" se delimitó con base en las siguientes provincias biogeográficas: Xerófila mexicana, Pacífica, Mesoamericana de Montaña, Caribe y Guajira, desde México hasta Nicaragua.

Para evaluar los modelos, se utilizó el área bajo la curva (Area Under Curve o AUC) de la característica operativa del receptor (Receiver Operating Characteristic o ROC), implementada directamente en Maxent. Asimismo, para complementar la evaluación de los modelos, se usó el método de ROC parcial (Peterson, Papes, \& Soberón, 2008) en el software propuesto por Barve (2008). El resultado probabilístico (0 a 1) de cada MNE obtenido se convirtió en un mapa binario de presencia-ausencia, cuyo valor de corte fue del $5 \%$ de presencias de entrenamiento. Por último, se realizó un análisis de MOP (Mobility Oriented Parity; Owens et al., 2013) con la finalidad de calcular la analogía climática que podría existir entre la "M" que se delimitó para el MNE del centro de origen y el espacio geográfico donde se proyectó, correspondiente al área de domesticación.

Comparación ambiental entre el centro de origen y el área de domesticación: Para comparar las condiciones ambientales entre el centro de origen y el de domesticación, se utilizaron las ocho variables con que se corrieron los MNE del tamaño (extensión) del área de accesibilidad o "M" previamente descritas. Posteriormente, se realizó la prueba propuesta por Broennimann et al. (2012), modificada por Di Cola et al. (2017). Esta propuesta se basa en un análisis multivariado (componentes principales) e incluye una primera prueba que es de equivalencia de nicho. En este caso particular, se evaluó si las condiciones ambientales en el centro de origen eran equivalentes (hipótesis nula) a las del área de domesticación o si eran menos equivalentes que lo esperado por azar (hipótesis alternativa, $\mathrm{P}<0.05$ ).
La segunda prueba realizada fue la de similitud de nicho, donde la hipótesis nula indica que los nichos son similares, mientras que la hipótesis alternativa indica que los nichos son menos similares que lo esperado por azar $(\mathrm{P}<0.05)$. En ambas pruebas, el azar o modelos nulos están representados por 100 repeticiones. Por ello, utilizamos la opción "lower" para determinar la hipótesis alternativa (sensu Broennimann et al., 2012, Di Cola et al., 2017). Finalmente, las variables climáticas que se utilizaron para correr los MNE del centro de origen y de domesticación fueron sometidas a un análisis de varianza y una prueba de comparación de medias (Tukey; $\mathrm{P} \leq 0.05$ ) para detectar si existían diferencias significativas entre ambos conjuntos de variables.

\section{RESULTADOS}

El MNE en el centro de origen tiene una extensión geográfica de $5217041 \mathrm{~km}^{2}$ y se distribuye en la vertiente del Pacífico en Suramérica desde Panamá hasta el norte de Chile, Argentina, Paraguay y el oeste de Brasil. Los valores de idoneidad más altos se ubicaron principalmente en Perú (Anexo 1). En el caso del área de domesticación, esta tuvo una extensión de $1084894 \mathrm{~km}^{2}$ que se distribuye desde la vertiente del Pacífico y el Golfo de México hasta Honduras. Los valores de idoneidad más altos se ubicaron principalmente en la vertiente del Golfo de México (Anexo 2).

Por su parte, la proyección del área de origen al de domesticación tuvo una extensión de $1002983 \mathrm{~km}^{2}$. De este total, las condiciones ambientales en $511618 \mathrm{~km}^{2}$ fueron parecidas a las del centro de origen, mientras que en el resto $\left(491365 \mathrm{~km}^{2}\right)$ las condiciones ambientales fueron diferentes. Por último, la proyección del área de domesticación a la de origen tuvo una extensión de $1481987 \mathrm{~km}^{2}$. De este total, las condiciones ambientales fueron parecidas en $392529 \mathrm{~km}^{2}$ a las del área de domesticación; en el resto $\left(1089458 \mathrm{~km}^{2}\right)$, las condiciones ambientales resultaron diferentes (Fig. 1A, Fig. 1B). Con base en las 27 localidades con presencia de $S$. $l$. var cerasiforme 
en el estado de Veracruz, se obtuvieron ocho registros en el norte, 60 en el centro y ocho en el sur, en diferentes tipos de vegetación y un amplio gradiente altitudinal y de variables ambientales (Tabla 1).

Los valores de AUC del MNE de origen y de domesticación fueron 0.93 y 0.80 respectivamente para los datos de entrenamiento. Los valores de ROC-parcial fueron 1.86 y 1.71 respectivamente $\mathrm{y}$, en ambos casos, fueron significativos $(\mathrm{P}=0.0001)$. Esto implica que el modelo al azar fue un buen modelo predictivo. Por otro lado, el resultado del análisis de MOP reveló que el área geográfica con climas no análogos entre las condiciones climáticas entre el centro de origen y área de domesticación fue mínima (Anexo 3). Por lo tanto, las proyecciones del MNE de origen al MNE de domesticación fueron adecuadas.

Con respecto a la comparación ambiental entre el centro de origen y de domesticación, el análisis de componentes principales mostró que el primer componente explicó el $57.42 \%$ de la variación total y las variables de temperatura (bio1, bio2, bio3, bio4) y de precipitación (bio14) fueron las más importantes. El segundo componente explicó solamente el $17.36 \%$ de la variación y el índice de variación estacional de la precipitación (bio15) fue lo más importante (Fig. 2A). También observamos que las condiciones ambientales del centro de origen presentan una mayor amplitud con respecto al área de domesticación (Fig. 2B, Fig. 2C, Fig. 2D). La hipótesis nula de equivalencia fue rechazada $(\mathrm{P}=0.003$, Fig. 2E), es decir, los modelos de nicho ecológico entre ambas regiones fueron menos equivalentes que lo esperado por azar. Por otro lado, en el caso de la prueba de similitud, no se rechazó la hipótesis nula $(\mathrm{P}=0.683$;
Fig. 2F). Esto nos indica que la similitud que existe entre ambas regiones también se puede obtener por azar.

Al comparar las variables del centro de origen contra el área de domesticación, se encontraron diferencias significativas $(\mathrm{P} \leq 0.05)$ en tres variables de temperatura (bio2, bio3, bio4) y tres variables de precipitación (bio12, bio14, bio15), pero no se detectaron diferencias significativas al comparar temperatura media anual (bio1) y la precipitación del trimestre más seco (bio18; Tabla 2). Cabe señalar que en el centro de origen llueve tres veces más (bio14) que en su área de domesticación, mientras que el índice de variación de temperatura (bio4) y de precipitación (bio15) en esta zona es menor en comparación con el área de domesticación. Esto indica que el clima en el centro de origen es más uniforme a lo largo del año, mientras que en el área de domesticación está sujeto a cierta variación ambiental.

Las medias con letras diferentes indican diferencias significativas $(\mathrm{P}<0.05)$. bio1: temperatura media anual $\left({ }^{\circ} \mathrm{C}\right)$, bio2: rango medio diurno de temperatura $\left({ }^{\circ} \mathrm{C}\right)$, bio3: isotermalidad, bio4: índice de variación estacional de temperatura, bio12: precipitación media anual $(\mathrm{mm})$, bio14: precipitación del mes más seco $(\mathrm{mm})$, bio15: índice de variación estacional de la precipitación, bio18: precipitación del trimestre más seco $(\mathrm{mm})$. Means with different letters indicate significant differences $(\mathrm{P}$ $<0.05)$. bio1: annual mean temperature ${ }^{\circ} \mathrm{C}$, bio2: mean diurnal range temperature ${ }^{\circ} \mathrm{C}$, bio3: isothermality, bio4: temperature seasonality, bio12: annual precipitation, bio14: precipitation of driest month (mm), bio15: precipitation seasonality, and bio18: precipitation of warmest quarter $(\mathrm{mm})$.

TABLA 2

ANOVA y prueba de Tukey entre las variables del centro de origen y de domesticación

TABLE 2

ANOVA and Tukey test between variables of origin and domestication regions

\begin{tabular}{lcccccccc} 
& bio1 & bio2 & bio3 & bio4 & bio12 & bio14 & bio15 & bio18 \\
Origen & $21.28^{\mathrm{a}}$ & $10.873^{\mathrm{b}}$ & $79.58^{\mathrm{a}}$ & $754.21^{\mathrm{b}}$ & $1768.73^{\mathrm{a}}$ & $74.01^{\mathrm{a}}$ & $49.35^{\mathrm{b}}$ & $445.31^{\mathrm{a}}$ \\
Domesticación & $22.24^{\mathrm{a}}$ & $13.326^{\mathrm{a}}$ & $61.73^{\mathrm{b}}$ & $2644.05^{\mathrm{a}}$ & $1275.28^{\mathrm{b}}$ & $20.75^{\mathrm{b}}$ & $87.85^{\mathrm{a}}$ & $485.22^{\mathrm{a}}$ \\
\hline
\end{tabular}




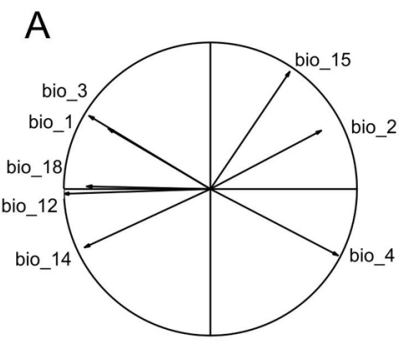

$\mathrm{PC} 1=57.42 \%, \mathrm{PC} 2=17.36 \%$

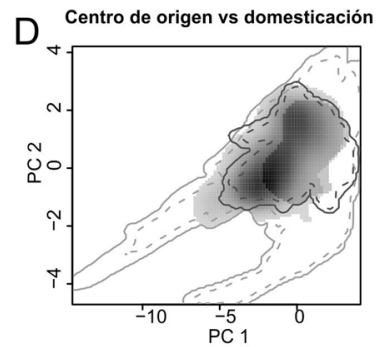

B

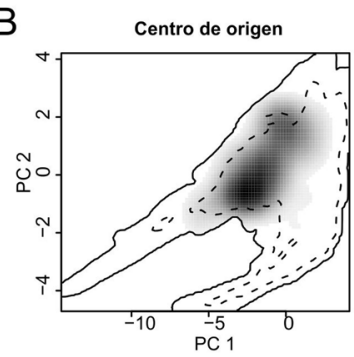

$E$

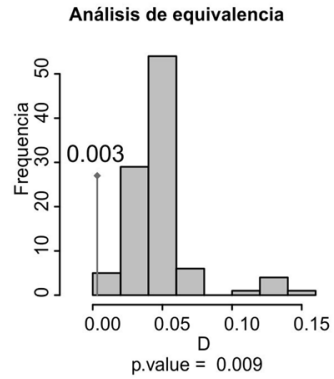

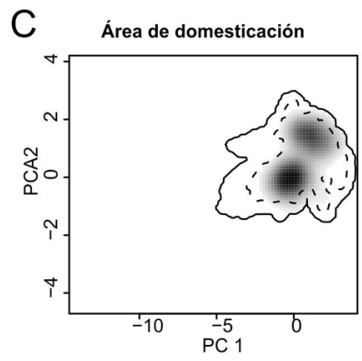

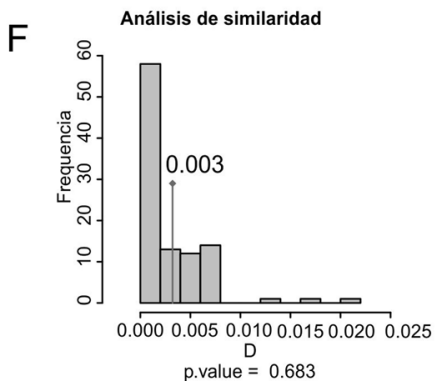

Fig. 2. Comparación de nicho ecológico de $S$. l. var. cerasiforme entre el centro de origen y el área de domesticación. En A se presenta la contribución de cada una de las variables para los primeros dos componentes principales (PC).

En B, C y D se presenta la amplitud ambiental de cada región (en términos de los dos primeros componentes). La línea continua de los polígonos representa el $100 \%$ de cada la región, mientras que la línea punteada el $50 \%$. En B y C, el sombreado en gris representa la densidad de las ocurrencias en cada región (en negro la densidad más alta). En D se presenta la amplitud ambiental de ambas regiones y su correspondiente superposición (en gris). En E se representa la prueba de equivalencia y en F la de similitud de nichos; las barras representan los modelos nulos mientras que el valor observado (D $=0.003$ ) está representado por una flecha. En ambos casos, el índice de similitud va de 0 a 1 , donde 0 es más diferente y 1 es más similar.

Fig. 2. Comparison of ecological niche of $S$. l. var. cerasiforme between the center of origin and the area of domestication. A shows the contribution of each of the variables for the first two main components (PC).

In $\mathrm{B}, \mathrm{C}$ and $\mathrm{D}$, we highlight the environmental amplitude of each region (in terms of the first two components). The continuous line of the polygons represents $100 \%$ of each region, the dotted line $50 \%$. In B and C, the shading in gray indicates the density of the occurrences in each region (in black the highest density). In D the environmental amplitude of both regions and their corresponding superposition (in gray) is presented. In E, we show the equivalence test and in $\mathrm{F}$ the of similarity test of niches. The bars represent the null models while the observed value $(D=0.003)$ is represented by an arrow. In both cases, the similarity index goes from 0 to 1 , where 0 is more different and 1 is more similar.

\section{DISCUSIÓN}

Los MNE y sus correspondientes transferencias entre el centro de origen y de domesticación de $S$. $l$. var cerasiforme mostraron áreas geográficas donde se superponen ambas regiones, por ejemplo, el centro de México. Esto quiere decir que existen poblaciones de tomate cuyos requerimientos ambientales son muy parecidos tanto en Suramérica como en México. Sin embargo, también se observaron áreas geográficas en México (domesticación) cuyas condiciones ambientales no fueron equivalentes a las de su centro de origen, específicamente el noroeste de México y la vertiente del Golfo de México, como se demostró en la prueba de comparación de nichos (Broennimann et al., 2012; Di Cola et al., 2017).

Cabe señalar que se tiene registro histórico de presencia de $S$. $l$. var cerasiforme en ambos casos, incluso en la vertiente del Golfo de México se corroboró su presencia actual durante el trabajo de campo, lo que sugiere su viabilidad. El hecho de encontrar poblaciones 
de tomate en el área de domesticación con distintas condiciones ambientales a las de su origen podría sugerir que existe un proceso de adaptación y variación genética para sobrevivir en entornos diferentes a los de su origen, particularmente si consideramos que el ambiente es uno de los factores que provocan cambios que se pueden manifestar a nivel morfológico y genético de una especie (Schluter, 2000). Esta relación entre las variables climáticas y diferencias genotípicas en poblaciones de otras especies de tomates ya ha sido reportada por otros autores (Zuriaga et al., 2009; Sifres. Blanca, \& Nuez, 2011).

Con respecto a las variables ambientales de temperatura, Vargas et al. (2005) registraron plantas de tomate a una temperatura media anual entre 18 y $22{ }^{\circ} \mathrm{C}$ en el área de domesticación; mientras que Nakazato, Bogonovich, \& Moyle (2008) reportaron una temperatura media anual de $20.8{ }^{\circ} \mathrm{C}$ en Suramérica. Los valores de temperatura media anual obtenidos en este trabajo $\left(21.28{ }^{\circ} \mathrm{C}\right.$ en su centro de origen y $22.24{ }^{\circ} \mathrm{C}$ en el área de domesticación) no presentaron diferencias significativas entre ambas regiones y coinciden con los resultados reportados previamente por otros autores. Esto sugiere que en el área de domesticación existen las condiciones de temperatura media anual para la distribución y desarrollo del tomate.

Por otro lado, en las variables de temperatura donde sí se encontraron diferencias significativas, el índice de variación estacional fue mayor para el área de domesticación. Esto implica una mayor fluctuación de temperatura a largo del año en comparación con el centro de origen. Aunado a lo anterior, el rango medio diurno de temperatura, que hace referencia a una fluctuación de temperatura entre el día y la noche, mostró que para el área de domesticación $\left(13{ }^{\circ} \mathrm{C}\right)$ fue $2{ }^{\circ} \mathrm{C}$ superior a la del centro de origen $\left(10.8^{\circ} \mathrm{C}\right)$. Esta variación de temperatura a diferentes escalas (estacional y durante el día y la noche) es importantes ya que esto afecta la floración, fructificación y crecimiento de la planta (Chaves-Barrantes \& Gutiérrez-Soto, 2017). Asimismo, la temperatura controla la germinación de la semilla del tomate (Labouriau \& Osborn, 1984), activando las reacciones enzimáticas que toman lugar durante el proceso de germinación de semillas de la planta (Liu et al., 2013).

Durante el trabajo de campo, pudimos observar algunas características como plantas con abundante follaje de color verde intenso, decoloración en los frutos- por ejemplo, frutos de color rojo con poca intensidad -, así como plantas con crecimiento restringido particularmente del tallo. Estas características podrían responder a la variación de la temperatura como ya lo han sugerido otros autores (Yang, Li, Li, Zhang \& Thomas, 2016; San MartínHernández, Ordaz-Chaparro, Sánchez-García, Beryl Colinas-Leon, \& Borges-Gómez, 2012). Sin embargo, estas características corresponden a observaciones generales de los ejemplares en campo, por lo que es necesario realizar un análisis formal y evaluar las características morfológicas tanto del centro de domesticación como del área de domesticación.

En el caso de las variables de precipitación, algunos reportes de precipitación media anual para el área de domesticación, como el de Vargas et al. (2005), indican que oscila entre 800 y 1000 mm. Mientras tanto, ÁlvarezHernández et al. (2009) muestra una oscilación similar entre 751 y $1014 \mathrm{~mm}$. No obstante, en el presente trabajo la precipitación media anual obtenida fue de $1275 \mathrm{~mm}$. Todos estos valores fueron menores a los reportados para el centro de origen: Nakazato et al. (2010) reporta 1800 $\mathrm{mm}$, por ejemplo. Es importante aclarar que estos datos contabilizaron la precipitación del mes más seco, ya que en el área de domesticación llueve menos $(20.75 \mathrm{~mm})$ con respecto al centro de origen $(74.01 \mathrm{~mm})$.

Así también, el índice de variación estacional es significativamente menor en el centro de origen en comparación con el área de domesticación. Esto quiere decir que, además de existir una mayor precipitación en el en centro de origen, la lluvia es constante a lo largo del año. Estas diferencias son muy importantes ya que la lluvia interviene en el transporte de nutrientes, fotosíntesis, mantenimiento de la turgencia e hidratación de las células de la planta 
(Basurto-Sotelo, Núñez, Pérez, \& Hernández, 2012), lo cual podría ser un indicador de diferenciación y adaptación de las poblaciones de $S$. $l$. var cerasiforme a nuevas condiciones de precipitación.

Por otro lado, los registros de $S$. $l$. var. Cerasiforme, específicamente en el estado de Veracruz, muestran cómo las poblaciones se encuentran en una variedad importe de tipos de vegetación desde zonas de manglar a baja altitud (14 m.s.n.m.) hasta bosques de encinos y coníferas (2 100 m.s.n.m.); la población pasa por áreas con cierto grado de perturbación como zonas agrícolas y ganaderas. También se obtuvieron registros en sitios con una variabilidad climática importante, por ejemplo, entre 1166 y $2260 \mathrm{~mm}$ de precipitación promedio anual y en intervalos de temperatura entre $5 \mathrm{y}$ $34{ }^{\circ} \mathrm{C}$. Este último dato es de particular importancia porque, bajo condiciones controladas, se sabe que una temperatura inferior a $12{ }^{\circ} \mathrm{C}$ limita el crecimiento y desarrollo de la planta (Hu, Zhou, Du, Xia, \& Yu, 2006). La presencia de poblaciones de $S$. $l$. var. cerasiforme en este amplio gradiente de condiciones, incluso bajo su límite de tolerancia como es el caso de la temperatura, pone en evidencia su plasticidad y amplia capacidad de adaptación como ya ha sido reportado en otros estudios (Comlekcioglu, Simsek, Boncuk, \& Aka-Kacar, 2010; Kamel, Soliman, Mandour, \& Ahmed, 2010; Flores-Hernández et al., 2017).

Es importante aclarar que es posible que, de los ejemplares colectados durante el trabajo de campo en Veracruz, haya plantas domesticadas o semi-domesticadas; sin embargo; no nos es posible determinar su grado de domesticación en el presente estudio. No obstante, consideramos que la comparación entre el centro de origen en Suramérica y el de domesticación en México es adecuado ya que se comparan las condiciones ambientales de ambas regiones y los lugares donde actualmente hay poblaciones de $S$. $l$. var. cerasiforme.

Finalmente, el centro de origen y de domesticación de $S$. $l$. var. cerasiforme tienen características ambientales en común a pesar de la distancia geográfica; no obstante, existen áreas en la región de domesticación con condiciones ambientales diferentes a las de su centro de origen, particularmente en la vertiente del Golfo de México en Veracruz. Los registros obtenidos en esta región se encuentran en un gradiente ambiental muy amplio que sugiere una plasticidad y amplia capacidad de adaptación del tomate a estos ambientes. Consideramos que las localidades de registro en esta región requieren de medidas de manejo y conservación, ya que podrían representar un material fundamental para los bancos de germoplasma.

\section{AGRADECIMIENTOS}

Se agradece al CONACyT por la beca otorgada al primer autor, a la Unidad de Manejo y Conservación de Recursos Genéticos de la Facultad de Ciencias Biológicas y Agropecuarias y al INECOL A. C. por facilitar la realización de este trabajo. En especial, se extienden agradecimientos a Alex Peña y Angelina Ruiz por su apoyo en el laboratorio y a Viridiana G. Ixtepan Guexpal por su apoyo en el trabajo de campo.

\section{RESUMEN}

El tomate (Solanum lycopersicum) es una de las principales hortalizas en el mundo debido a las importantes ganancias económicas que genera su comercialización; sin embargo, a pesar de que la especie es ampliamente cultivada en el mundo, su diversidad genética se considera restringida. Esto hace que su resistencia a plagas y enfermedades en las variedades actualmente cultivadas sea baja. Existe la forma silvestre $S$. $l$. var ceraciforme que se distribuye desde Suramérica (centro de origen) hasta México (área de domesticación), específicamente en la vertiente del Golfo de México en Veracruz. Como objetivo, se quiso identificar patrones de diversificación ambiental con los que se pudiesen inferir procesos de adaptación de las poblaciones en el área de domesticación con respecto a su centro de origen. Asimismo, se planteó proponer medidas de conservación y rescate del tomate $S$. $l$. var. cerasiforme. Se realizaron modelos de nicho ecológico (MNE) con Maxent versión 3.4.1 (evaluados con AUC y ROC-parcial) para determinar la idoneidad de las condiciones ambientales, incluyendo proyecciones del centro de domesticación al área de origen y viceversa. Esto se realizó con base en ocho variables de temperatura y precipitación. Los registros de tomate fueron tomados de fuentes bibliográficas y trabajo de campo. 
Seguidamente, se realizó la prueba de comparación de nichos (equivalencia y similitud), propuesta por Broennimann et al. (2012), para evaluar la similitud de condiciones ambientales en ambas regiones. Posteriormente, se realizó un análisis de varianza seguido de una prueba de comparación de medias (Tukey, $\mathrm{P} \leq 0.05$ ) con las ocho variables ambientales utilizadas. Los valores de AUC (0.93 y 0.80$)$ y ROC-parcial obtenidos (1.86 y $1.71 ; \mathrm{P}=0.0001)$ de los MNE muestran que fueron buenos modelos predictores. Se observó que en el centro de México existen condiciones ambientales similares a las del centro de origen, a diferencia de la vertiente del Golfo de México donde son diferentes. La prueba de equivalencia mostró que la comparación ambiental entre ambas regiones es menos equivalente que lo esperado por azar $(\mathrm{P}=0.003)$. Mientras tanto, la otra prueba indica que la similitud que existe entre ambas regiones también se puede obtener por azar $(\mathrm{P}=0.683)$. También, se encontraron diferencias significativas en tres variables de temperatura y precipitación. En conclusión, el centro de origen y de domesticación de $S$. $l$. var. cerasiforme tienen características ambientales en común a pesar de la distancia geográfica, pero existen zonas geográficas (vertiente del Golfo de México en Veracruz) en el área de domesticación con condiciones ambientales diferentes a las de su centro de origen y con un potencial importante como bancos de germoplasma.

Palabras clave: distribución; modelado de nicho ecológico; predictores ambientales; centro de origen, área de domesticación; comparación de nicho ecológico.

\section{REFERENCIAS}

Álvarez-Hernández, J. C., Cortez-Madrigal, H., \& GarcíaRuiz, I. (2009). Exploración y caracterización de poblaciones silvestres de jitomate (Solanaceae) en tres regiones de Michoacán, México. Polibotánica, $28,139-159$.

Arce, P. G. (2015). Distribución geográfica de los tomates silvestres (Solanum 1. sect. lycopersicon (mill.) wettst.: solanaceae). Arnaldoa, 20, 301-314.

Barve, N. (2008). Tool for partial-ROC. Version 1. Retrieved from https://www.scienceopen.com/ document?vid=8a2f903 1 -f73f-47b7-a043 b3c680d5c5d1

Basurto-Sotelo, M., Núñez, B. A., Pérez, L. R., \& Hernández, R. O. (2012). Estrés ambiental en plantas. Revista Chapingo, Serie Ciencias Forestales y del Ambiente, 54, 1-8.

Bolger, A. M., Lohse, M., \& Usadel, B. (2014). Trimmomatic: a flexible trimmer for Illumina sequence data. Bioinformatics, 30, 2114-2120.

Bonilla-Barrientos, O., Lobato-Ortiz, R., García-Zavala, J. J., Cruz-Izquierdo, S., Reyes-López, D.,
Hernández-Leal, E., \& Hernández-Bautista, A. (2014). Diversidad agronómica y morfológica de tomates arriñonados y tipo pimiento de uso local en Puebla y Oaxaca, México. Revista Fitotecnia Mexicana, 37, 129-139.

Broennimann, O., Fitzpatrick, M. C., Pearman, P. B., Petitpierre, B., Pellissier, L., Yoccoz, N. G., \& Graham, C. H. (2012). Measuring ecological niche overlap from occurrence and spatial environmental data. Global Ecology and Biogeography, 21, 481-497.

Chaves-Barrantes, N. F., \& Gutiérrez-Soto, M. V. (2017). Respuestas al estrés por calor en los cultivos. II. Tolerancia y tratamiento agronómico. Agronomía Mesoamericana, 28, 255-271.

Comlekcioglu, N., Simsek, O., Boncuk, M., \& Aka-Kacar, Y. (2010). Genetic characterization of heat tolerant tomato (Solanum lycopersicon) genotypes by SRAP and RAPD markers. Genetics and Molecular Research, 9, 2263-2274.

Croteau, E. K. (2010). Causes and consequences of dispersal in plants and animals. Nature Education Knowledge, 3(10), 12 .

Di Cola, V., Broennimann, O., Petitpierre, B., Breiner, F. T., D'amen, M., Randin, C., \& Pellissier, L. (2017). ecospat: an R package to support spatial analyses and modeling of species niches and distributions. Ecography, 40, 774-787.

Elith, J., Phillips, S. J., Hastie, T., Dudík, M., Chee, Y. E., \& Yates, C. J. (2011). A statistical explanation of MaxEnt for ecologists. Diversity and Distributions, $17,43-57$.

Escobar, L. E., Qiao, H., Phelps, N. B., Wagner, C. K., \& Larkin, D. J. (2016). Realized niche shift associated with the Eurasian charophyte Nitellopsis obtusa becoming invasive in North America. Scientific Reports, 6, 29037.

FAOSTAT. FAO statistical program of work (2016) country indicators, crop statistic. Retrieved from http://www. fao.org/faostat/en/\#data/QC

Flores-Hernández, L. A., Lobato-Ortiz, R., García-Zavala, J. J., Molina-Galán, J. D., Sargerman-Jarquín, D. M., \& Velasco-Alvarado, M. D. J. (2017). Parientes silvestres del tomate como fuente de germoplasma para el mejoramiento genético de la especie. Revista Fitotecnia Mexicana, 40, 83-91.

Global Biodiversity Information Facility. (2018). Registros de Solanum lycopersicum var. cerasiforme. Recuperado de https://doi.org/10.15468/dl.emrmds

Govindaraj, M., Vetriventhan, M., \& Srinivasan, M. (2015). Importance of genetic diversity assessment in crop plants and its recent advances: an overview of its analytical perspectives. Genetics Research International, 431487, 1-14. 
Hijmans, R. J., Cameron, S. E., Parra, J. L., Jones, P. G., \& Jarvis, A. (2005). Very high resolution interpolated climate surfaces for global land areas. International Journal of Climatology, 25, 1965-1978.

Hoyt, E. (1992). Conservando los parientes silvestres de las plantas cultivadas. Wilmington, EUA: Consejo Internacional de Recursos Fitogenéticos.

Hu, W. H., Zhou, Y. H., Du, Y. S., Xia, X. J., \& Yu, J. Q. (2006). Differential response of photosynthesis in greenhouse-and field-ecotypes of tomato to longterm chilling under low light. Journal of Plant Physiology, 163, 1238-1246.

Jenkins, J. A. (1948). The origin of the cultivated tomato Economic Botany, 2, 379-392.

Kamel, M. A., Soliman, S. S., Mandour, A. E., \& Ahmed, M. S. (2010). Genetic evaluation and molecular markers for heat tolerance in tomato (Lycopersicon esculentum Mill.). Journal of American Science, 6 , 364-374.

Khimoun, A., Cornuault, J., Burrus, M., Pujol, B., Thebaud, C., \& Andalo, C. (2013). Ecology predicts parapatric distributions in two closely related Antirrhinum majus subspecies. Evolutionary Ecology, 27, 51-64.

Labouriau, L. G., \& Osborn, J. H. (1984). Temperature dependence of the germination of tomato seeds. Journal of Thermal Biology, 9, 285-294.

Liu, K., Baskin, J. M., Baskin, C. C., Bu, H., Du, G., \& Ma M. (2013). Effect of diurnal fluctuating versus constant temperatures on germination of 445 species from the eastern Tibet Plateau. PloS one, 8(7), e69364.

Long, J. (1995). De tomates y jitomates en el siglo XVI. Estudios de Cultura Náhuatl, 25, 239-252.

Löwenberg-Neto, P. (2014). Neotropical region: a shapefile of Morrone's (2014) biogeographical regionalization. Zootaxa, 3802, 300.

Lozier, J. D., Aniello, P., \& Hickerson, M. J. (2009). Predicting the distribution of Sasquatch in western North America: anything goes with ecological niche modelling. Journal of Biogeography, 36, 1623-1627.

Morrone, J. J. (2014). Biogeographical regionalisation of the Neotropical region. Zootaxa, 3782, 1-110.

Nakazato, T., Bogonovich, M., \& Moyle, L. C. (2008). Environmental factors predict adaptive phenotypic differentiation within and between two wild Andean tomatoes. Evolution, 62, 774-792.

Nakazato, T., Warren, D. L., \& Moyle, L. C. (2010). Ecological and geographic modes of species divergence in wild tomatoes. American Journal of Botany, 97, 680-693.
Owens, H. L., Campbell, L. P., Dornak, L. L., Saupe, E. E., Barve, N., Soberón, J., \& Peterson, A. T. (2013). Constraints on interpretation of ecological niche models by limited environmental ranges on calibration areas. Ecological Modelling, 263, 10-18.

Pearson, R. G., \& Dawson, T. P. (2003). Predicting the impacts of climate change on the distribution of species: are bioclimate envelope models useful. Global Ecology and Biogeography, 12, 361-371.

Peralta, I. E., Knapp, S., \& Spooner, D. M. (2005). New species of wild tomatoes (Solanum section Lycopersicon: Solanaceae) from Northern Peru. Systematic Botany, 30, 424-434.

Peralta, I. E., \& Spooner, D. M. (2007). History, origin and early cultivation of tomato (Solanaceae). In M. K. Razdan \& A. K. Mattoo (Eds.), Genetic improvement of solanaceous crops (pp. 1-24). New Hampshire, USA: Science Publishers.

Peterson, A. T., Papeş, M., \& Soberón, J. (2008). Rethinking receiver operating characteristic analysis applications in ecological niche modeling. Ecological Modelling, 213, 63-72.

Phillips, S. J., \& Dudík, M. (2008). Modeling of species distributions with Maxent: new extensions and a comprehensive evaluation. Ecography, 31, 161-175.

Phillips, S. J., Anderson, R. P., \& Schapire, R. E. (2006). Maximum entropy modeling of species geographic distributions. Ecological Modelling, 190, 231-259.

Ranc, N., Muños, S., Santoni, S., \& Causse, M. (2008). A clarified position for Solanum lycopersicum var. cerasiforme in the evolutionary history of tomatoes (Solanaceae). BMC Plant Biology, 8, 130-145.

Rao, V. R., \& Hodgkin, T. (2002). Genetic diversity, conservation, and utilization of plant genetic resources. Plant Cell, Tissue and Organ Culture, 68, 1-19.

Sánchez-Peña, P., Oyama, K., Núñez-Farfán, J., Fornoni, J., Hernández-Verdugo, S., Márquez-Guzmán, J., \& Garzón-Tiznado, J. A. (2006). Sources of resistance to whitefly (Bemisia spp.) in wild populations of Solanum lycopersicum var. cerasiforme (Dunal) spooner GJ Anderson et RK Jansen in Northwestern Mexico. Genetic Resources and Crop Evolution, 53, 711-719.

San Martín-Hernández, S., Ordaz-Chaparro, V. M., Sánchez-García, P., Beryl Colinas-Leon, M., \& BorgesGómez, L. (2012). Calidad de tomate (Solanum lycopersicum L.) producido en hidroponía con diferentes granulometrías de tezontle. Agrociencia, 46, 243-254.

Schluter, D. (2000). Ecological character displacement in adaptive radiation. The American Naturalist, 156, S4-S16. 
Schoener, T. W. (1968). The Anolis lizards of Bimini: resource partitioning in a complex fauna. Ecology, 49, 704-726.

Sifres, A., Blanca, J., \& Nuez, F. (2011). Pattern of genetic variability of Solanum habrochaites in its natural area of distribution. Genetic Resources and Crop Evolution, 58, 347-360.

Soberón, J., \& Peterson, A. T. (2005). Interpretation of models of fundamental ecological niches and species'distributional areas. Biodiversity Informatics, $2,1-10$.

Vargas, C. D., Rodríguez, E. G., Sánchez, J. J. G., Montes, S. H., Ruiz, A. C., Lápiz, R. I., Puente, P. O., \& Martínez, J. L. R. (2005). Adaptación climática de Lycopersicum en el occidente de México. En S. Carvajal \& E. Pimienta Barrios, Avances en la Investigación
Cientifica en el CUCBA (pp. 207-210). Guadalajara, México: Universidad de Guadalajara.

Yang, Z., Li, Y., Li, P., Zhang, F., \& Thomas, B. W. (2016). Effect of difference between day and night temperature on tomato (Lycopersicon esculentum Mill.) root activity and low molecular weight organic acid secretion. Soil Science and Plant Nutrition, 62, 423-431.

Yang, X. Q., Kushwaha, S. P. S., Saran, S., Xu, J., \& Roy, P. S. (2013). Maxent modeling for predicting the potential distribution of medicinal plant, Justicia adhatoda $L$. in Lesser Himalayan foothills. Ecological Engineering, 51, 83-87.

Zuriaga, E., Blanca, J. M., Cordero, L., Sifres, A., BlasCerdán, W. G., Morales, R., \& Nuez, F. (2009). Genetic and bioclimatic variation in Solanum pimpinellifolium. Genetic Resources and Crop Evolution, 56, 39-51.

\begin{abstract}
ANEXO 1
Idoneidad de MNEorigen: los colores oscuros (negro) indican áreas de mayor idoneidad y los claros indican menor idoneidad
\end{abstract}

\title{
APPENDIX 1
}

MNEorigen suitability: dark colors (black) indicate areas of higher suitability and light colors indicate lower suitability

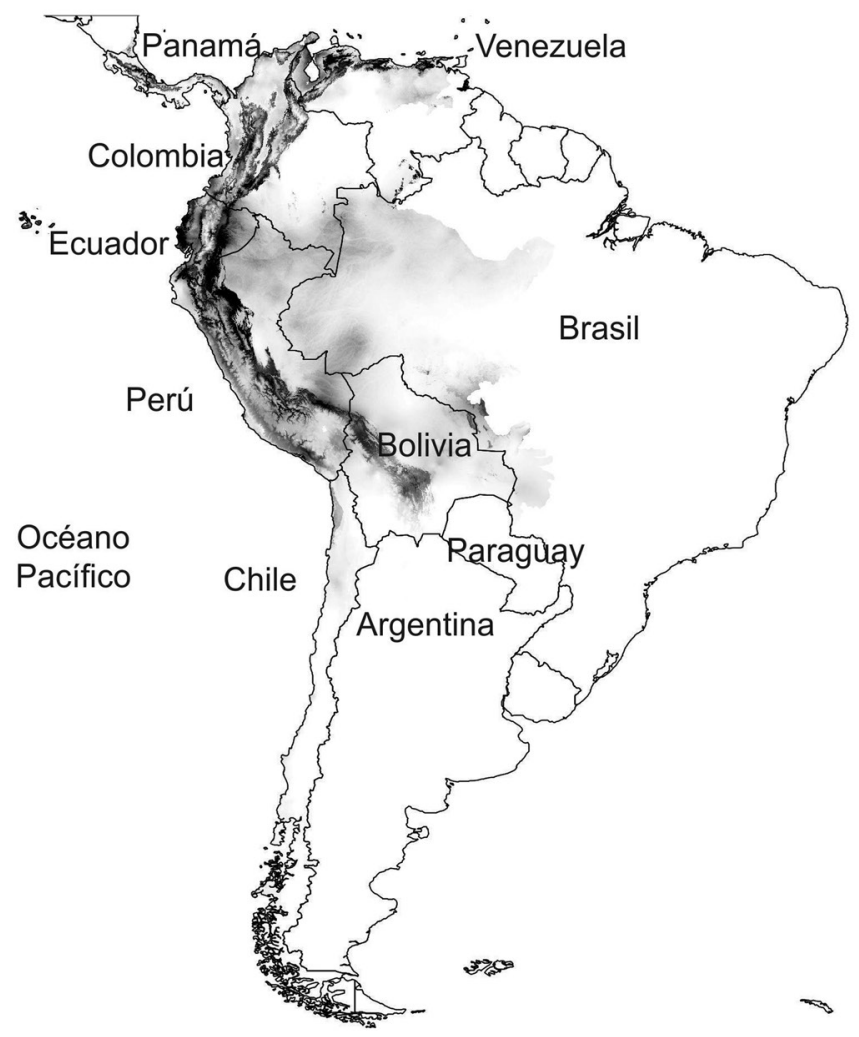




\section{ANEXO 2}

Idoneidad de MNEMéxico: los colores más oscuros indican áreas de mayor idoneidad y los claros indican menor idoneidad. Los polígonos representan los límites políticos; en el caso de México, se presentan los límites estatales

\section{APPENDIX 2}

MNEMéxico suitability: darker colors indicate areas of greater suitability and light colors indicate lower suitability. The polygons represent the political limits; in the case of Mexico, the state limits are presented.

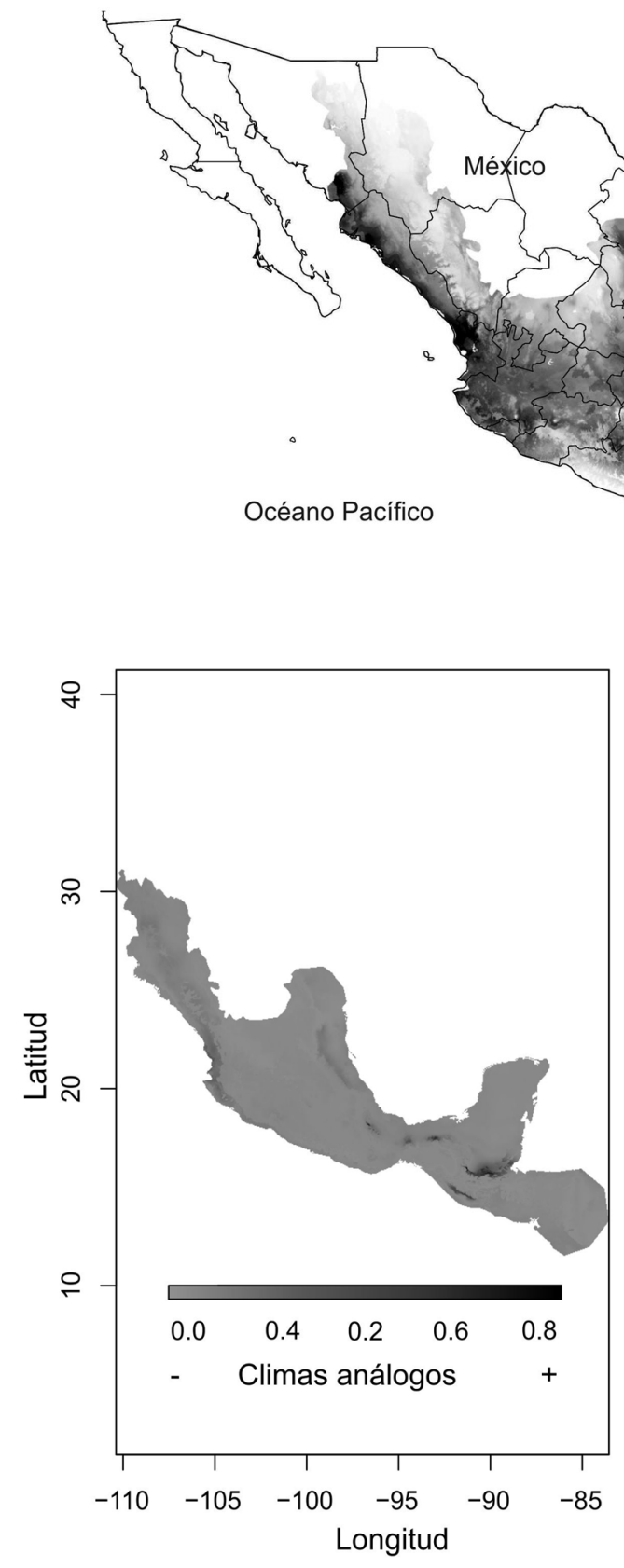

ANEXO 3

Climas análogos (colores claros) y climas no análogos (colores en oscuro)

\section{APPENDIX 3}

Analogous climates (light colors) and non-analogous climates (dark colors) 